\title{
The new oral anticoagulants: results of clinical trials and practical applications
}

\author{
Jack Ansell
}

Published online: 18 July 2013

(C) Springer Science+Business Media New York 2013

The coumarin-type oral anticoagulants or vitamin $\mathrm{K}$ antagonists (VKA) have been in use since the early 1940s. Their discovery evolved from investigations into a hemorrhagic disease of cattle in the early twentieth century attributed to the consumption of spoiled sweet clover [1]. Link, a biochemist at the University of Wisconsin, isolated the responsible agent in spoiled sweet clover, dicoumarol(3-3' methyl-bis-4-hydroxy coumarin), which quickly entered the clinical arena in 1941. Link subsequently synthesized a related compound (warfarin), initially popularized as a rodenticide in the late 1940s, that entered clinical practice in the 1950s and quickly became the major oral anticoagulant in clinical use. Little has changed in the formulation of the coumarin-type oral anticoagulants. They have remained critically important drugs in the primary and secondary prevention of thromboembolism. Despite their widespread use, the vitamin $\mathrm{K}$ antagonists suffer from a number of problems that lead to underuse and inadequate treatment.

Warfarin is safe and effective if a therapeutic international normalized ratio (INR) is properly maintained. In practice, however, it is difficult to manage because its therapeutic level is affected by many factors including diet, medications, illnesses, and genetics [2]. Warfarin's narrow therapeutic range predisposes to many adverse events from both under anticoagulation (resulting in thrombus formation) and over anticoagulation (leading to hemorrhage). To ensure that a therapeutic INR is achieved, frequent monitoring is required that may be inconvenient for patients and physicians and costly for the healthcare system. Additional warfarin drawbacks include its slow onset and offset of

J. Ansell (ه)

New York University School of Medicine, New York, NY, USA

e-mail: ansellje@gmail.com action. It takes 72-96 $\mathrm{h}$ to become effective and requires overlap with a rapidly acting parenteral anticoagulant or "bridging" until a therapeutic INR is achieved. Its slow offset, with an effective half-life of approximately $40 \mathrm{~h}$, makes it difficult to manage before procedures. Safety concerns and therapeutic complexity lead many physicians to underuse warfarin, prescribing it to only two-thirds of appropriate candidates [3].

Over the last decade a number of new oral anticoagulants have been developed and are now emerging from Phase III clinical trials [4]. Many of these agents are targeted to interact directly with, and inhibit a specific coagulation factor. This is in contrast to the VKAs that work indirectly by interfering with the synthesis of functional vitamin K-dependent coagulation proteins, thus, reducing their plasma concentration. Although commonly referred to as new or novel oral anticoagulants (NOACs), these drugs will quickly lose their newness and novelty. A more appropriate designation that denotes their mechanism of action (vis a vis VKAs) is "target-specific oral anticoagulants" or TSOAs. This designation will be used interchangeably with NOACs throughout this special issue.

The target-specific oral anticoagulants in development are free from many of warfarin's drawbacks and offer a convenient alternative to warfarin. Direct thrombin and direct Xa inhibitors are currently the most promising agents. By inhibiting these factors in the final common pathway of coagulation, these agents block both the intrinsic and extrinsic pathways. These small molecules directly block either factor Xa or IIa (thrombin) at their catalytic pocket. They promise to be more convenient and possibly safer than warfarin because they are given in fixed doses, have a predictable anticoagulant effect, do not require monitoring, have few or minimal interactions with drugs or diet, and have a rapid onset of action that eliminates the need for 
parenteral anticoagulation. Several landmark studies are now available for the direct thrombin inhibitor, dabigatran etexilate, and the two factor Xa inhibitors, rivaroxaban and apixaban, leading to the approval of these agents in the US for an increasing number of indications.

\section{Clinical implications}

How will clinicians translate the emerging data, the pros and the cons of the target-specific oral anticoagulants, into everyday, real world use [5]? At first, with limited indications, the impact of new agents may be minimal and will depend on a physician's level of comfort in using such agents. A patient selection process may eventually evolve based on real world experience since most of the phase III trials enrolled highly selected patients. Warfarin will also remain as the mainstay of treatment for patients with mechanical heart valves, since limited studies in this population have so far not proved successful [6]. Warfarin may also hold favor with patients who are considered noncompliant with therapy, and as an option for those patients that "fail" or develop an event while on one of the new agents. Lastly, patients on warfarin who are clinically defined as "non-responders" or who have difficulty maintaining a stable INR on warfarin may be ideal candidates for a new oral anticoagulant, but only if the INR instability is not due to poor compliance. Certainly, convenience of therapy will be a major motivating factor to change to a new, non-monitored anticoagulant drug.

Conflict of Interest Dr. Ansell is consultant for Bristol Myers Squibb, Pfizer, Janssen, Boehringer Ingelheim, Daiichi Sankyo, Portola.

\section{References}

1. Link KP (1959) The discovery of dicumarol and its sequels. Circulation 19:97-107

2. Ansell J, Hirsh J, Hylek E, Jacobson A, Crowther M, Palareti G (2008) The pharmacology and management of the vitamin K antagonists: ACCP evidence-based clinical practice guidelines. Chest 133:160S-198S

3. Birman-Deych E, Radford MJ, Nilasena DS, Gage BF (2006) Use and effectiveness of warfarin in Medicare beneficiaries with atrial fibrillation. Stroke 37:1070-1074

4. Weitz JI, Eikelboom JW, Samama MM (2012) New antithrombotic drugs: antithrombotic therapy and prevention of thrombosis, 9th ed: American College of Chest Physicians Evidence-Based Clinical Practice Guidelines. Chest 141(2_suppl):e120S-e151S. doi:10.1378/chest.11-2294

5. Ansell J (2011) New oral anticoagulants should not be used as first line agents to prevent thromboembolism in patients with atrial fibrillation. Circulation 125:165-170

6. Dabigatran RE-ALIGN phase 2 mechanical-valve trial partly halted. http://www.theheart.org/article/1462273.do. Accessed 14 June 2013 